\title{
El índice de orientación agrícola (ioa) como mecanismo de análisis del gasto público agropecuario Ecuatoriano
}

\section{The agricultural orientation index (aoi) as a mechanism of analysis in the public agricultural Ecuadorian expenditure}

Jean Pierre Yannuzzelli Rivadeneira

Víctor Quinde Rosales

Rina Bucaram Leverone

Universidad Agraria del Ecuador, Ecuador

Autor para correspondencia: jeanpierreyannu@ hotmail.com; vquinde@uagraria.edu.ec; rbucaram@uagraria.edu.ec

Fecha de recepción: 12 de Octubre 2017 - Fecha de aceptación: 15 de Febrero de 2018

Resumen: La presente investigación se basa en un razonamiento inductivo, el cual evaluó la incidencia del gasto público frente al sector agropecuario ecuatoriano, basándose en la aplicación del Índice de Orientación Agrícola, con el fin de deducir cuán importante es el sector agropecuario para el Gobierno. El sector agropecuario es una parte importante dentro de la economía ecuatoriana, la cual ha sido la actividad que permitió el desarrollo del sistema económico y precursora para la creación de las pocas industrias nacionales que existen en la actualidad. El gasto público agropecuario del Ecuador entre 2005 y 2015 presenta una tendencia positiva en términos relativos, gracias a la obtención del Índice de Orientación Agrícola afirmamos que dicho incremento no es el adecuado comparando la aportación que realiza el sector agropecuario al producto interno bruto, presentando un comportamiento distinto, con un resultado tan bajo en comparación a la unidad. Con la obtención del índice se confirma que, para Ecuador, el sector agropecuario no es un eje prioritario, otorgando un gasto público sin un previo estudio ni análisis de las necesidades del sector agropecuario, provocando un retraso en su desarrollo y productividad. Palabras Claves: índice de orientación agrícola; gasto público nacional; gasto publico agropecuario; producto interno bruto; producto interno bruto agropecuario; sistema económico; sector prioritario

Abstract: This investigation is based on an inductive reasoning, which evaluated the incidence of public expenditure in the Ecuadorian agricultural sector, based on the application of the Agricultural Orientation Index, in order to deduce how important is the agricultural sector for the Government. The agricultural sector is an important part of the Ecuadorian economy, this activity has allowed the development of the economic system and as been the precursor for the creation of the few national industries that exist today. Ecuador's agricultural public expenditure between 2005 and 2015 shows a positive trend in relative terms, thanks to obtention of the Agricultural Orientation Index, we affirm that the growth is not adequate, comparing with the contribution made by the agricultural sector to the gross domestic product, with a result so low in comparison to the unit. The results of the index confirmed that for Ecuador, the agricultural sector is not a 
priority axis, giving a public expenditure without a previous study or analysis of the needs of the agricultural sector, causing a delay in its development and productivity.

Key Words: agricultural orientation index; national public expenditure; agricultural public expenditure; gross domestic product; gross agricultural domestic product; priority sector

\section{Introducción}

La agricultura en Ecuador guarda relación con el proceso independentista del territorio ecuatoriano del yugo español y el nacimiento de una república con una dependencia extrema a este sector, gracias a su impulso productivo y comercial siendo inicialmente el producto insignia el cacao, producto que en tiempos de colonia tenía limitaciones institucionales generadas por España la cual buscaba un monopolio. Teorías como la de David Ricardo promovieron en América Latina un libre comercio en los puertos Iberoamericanos, concibiendo la idea en Guayaquil de crear una nación desde su puerto (Naranjo, 2009).

El sector agrícola es de importancia para el desarrollo económico del país entendiendo que mencionado sector no se encuentra aislado del resto de sectores. Hirschman (1958), Arias et al. (2005) ratifican lo expuesto manifestando que la agricultura es un ente de desarrollo económico importante para el resto de sectores.

Quinde (2015) menciona que la agricultura no es solo una actividad primaria productiva, el gobierno ecuatoriano debe interesarse en el sector como un activo estratégico. Yannuzzelli (2017) afirma que el sector agrícola es un eje vinculante para las economías en desarrollo. Echeverri et al. (2002) citado por FAO (2004) describe que la agricultura tiene la capacidad de abordar dimensiones económicas y no económicas, y que estas últimas son prioritarias actualmente ya que la agricultura manifiesta un conjunto de externalidades generando beneficios o perjuicios a la actividad productiva y que se escapan de la misma pero que afectan al conjunto social.

El actualmente nombrado Ministerio de Agricultura y Ganadería - MAG expone al sector agropecuario como el proveedor del el $97 \%$ del consumo alimenticio básico, asegurando la alimentación de 16 millones de personas, El Ecuador es autosuficiente en la producción agroalimentaria; con tan solo el 2,7\% de los alimentos importados donde, el 2,6\% son primarios y el $0,1 \%$ son industrializados (2016). Quinde (2011) entiende que el estado ecuatoriano asumir que el sector agrícola guarda importancia para el país en términos de producción para el consumo interno y su contribución a la economía gracias a las exportaciones.

El Instituto Nacional de Estadística y Censos - INEC expone que en la formación de indicadores laborales generados por la encuesta nacional de empleo, desempleo y subempleo ENEMDU del primer semestre del 2017 el 29\% de la población económicamente activa - PEA nacional y el $82 \%$ del PEA rural es parte de la actividad agricola, ganadera, silvícola y pesquera; entendiendo que esta actividad es primordial fuente de empleo e ingresos para la población rural y principalmente para la agricultura familiar campesina (2017).

La agricultura como tal integra un importante porcentaje de la población rural denominado pequeño productor familiar campesino sobre la cual el estado ecuatoriano ha manifestado tener una “deuda histórica social". Martínez (2009) describe que dentro de los 
espacios rurales se generan dinámicas que desarrollan los productores a pesar de tener factores adversos entendiendo a estos como apertura de mercados, dolarización, elevado costo de mano de obra, desinterés del estado y de los gobiernos locales.

Yannuzzelli et al. (2017) describe al gasto público como la inversión realizada por el gobierno en búsqueda del desarrollo en el país, donde es prioridad las necesidades de los sectores que componen la economía, obligaciones inmediatas del gobierno como programas y proyectos los cuales generen un beneficio a la sociedad y a sus sectores económicos. La FAO (2007) manifiesta que el gasto público es el esfuerzo de un país por atender necesidades nacionales prioritarias y financiar programas fundamentales de la política de desarrollo. Barcelata (2007) expone que el gasto público es un factor para el impulso y desarrollo de la economía y garantiza el bienestar social, ante esto existe la necesidad de incrementarlo, tal como lo hacen los países más desarrollados.

Hall (2010) ratifica esta idea al manifestar que a pesar de que el gasto público es considerado como una carga para una economía de mercado, la cual crecería más rápido si se limitara mencionado gasto, la historia demuestra lo contrario desde mediados del siglo XIX el crecimiento económico ha presentado una estrecha relación con el incremento proporcional del gasto público. Guereña (2011) argumenta al gasto público como la expresión más clara de la voluntad política asumiendo que la elaboración y ejecución del presupuesto del estado implica asignar prioridades entre las diferentes necesidades de inversión que tienen los sectores los cuales compiten por recursos limitados. Quinde (2011) corrobora esta idea manifestando que el estado ecuatoriano debe considerar la importancia que tiene el sector agrícola en términos de producción para el consumo interno y de su contribución a la economía con las exportaciones antes de beneficiar a otros sectores económicos del país.

Para el desarrollo del presente estudio se plantea el uso del Índice de Orientación Agrícola - IOA para establecer el aporte que genera el estado al sector agrícola en referencia al aporte que genera este sector al país. El documento evaluara el comportamiento del gasto público y del Producto Interno Bruto - PIB y su participación en el sector agropecuario de forma individual, previa al uso y análisis del IOA en el Ecuador.

\section{Metodología}

Para el desarrollo del artículo se presenta un tipo de razonamiento inductivo mediante el cual, el uso del indicador denominado IOA permitió evaluar el aporte porcentual que da el gasto público al sector agrícola en función de la contribución porcentual que este genera al PIB. Se esboza como proceso de desarrollo de la investigación un proceso unimétodo con un paradigma empírico-analítico según Bacon (1949) citado por Duque (2015) permite reflejar la realidad de la forma más fiel y neutral posible de la investigación realizada.

El diseño de investigación del documento estableció una base de datos de serie de tiempo anual con un periodo de evaluación del 2005 al 2015 de las variables PIB Total ${ }^{1}$, PIB Agrícola ${ }^{2}$, Gasto Público Total y Gasto Público Agrícola, se extrajo la información de la página del Banco

\footnotetext{
${ }^{1}$ PIB real con año base 2007
}

${ }^{2}$ PIB real con año base 2007 
Central del Ecuador. El Indicé de Orientación Agrícola como mecanismo para determinar la contribución existente del gasto público al sector agrícola, define su indicador.

$$
\text { IOA }=\frac{\frac{G P A}{\mathrm{GPT}}}{\frac{\mathrm{PIBA}}{\mathrm{PIBT}}}
$$

GPA es el Gasto Público Agrícola, GPT es el Gasto Público Total o Nacional, PIBA es el Producto Interno Bruto Agrícola y PIBT es el Producto Interno Bruto Total o Nacional.

\section{Resultados y discusión}

El gasto público del Ecuador ha presentado una variación irregular a lo largo de la última década con una tendencia positiva. Este comportamiento es similar en los desembolsos generados para el desarrollo del sector agropecuario el cual ha tenido fluctuaciones con tendencia al alza, pero con una participación porcentual mínima del total del egreso estatal (Tabla N¹).

Tabla 1. Participación Del Gasto Público Agrícola Dentro Del Gasto Público Total. (2005-

\begin{tabular}{|cccccccc}
\multicolumn{7}{c}{ 2015) } \\
\hline Ejercicio & $\begin{array}{c}\text { Gasto Publico (millones de } \\
\text { dólares) }\end{array} 2005-2015$ & $\begin{array}{c}\text { Gasto Publico (millones de } \\
\text { dólares) sector } \\
\text { Agropecuario 2005- 2015 }\end{array}$ & $\begin{array}{c}\text { Participación del sector } \\
\text { Agropecuario }\end{array}$ & $\begin{array}{c}\text { Variación Anual Gasto } \\
\text { Publico }\end{array}$ & $\begin{array}{c}\text { Variación Anual Gasto } \\
\text { Publico Agropecuaria }\end{array}$ \\
\hline 2005 & $\$$ & $7.914,71$ & $\$$ & 164,71 & $2 \%$ & - & - \\
2006 & $\$$ & $9.617,65$ & $\$$ & 119,48 & $1 \%$ & $22 \%$ & $-27 \%$ \\
2007 & $\$$ & $10.015,07$ & $\$$ & 123,62 & $1 \%$ & $4 \%$ & $3 \%$ \\
2008 & $\$$ & $13.838,90$ & $\$$ & 245,50 & $2 \%$ & $38 \%$ & $99 \%$ \\
2009 & $\$$ & $14.200,80$ & $\$$ & 430,03 & $3 \%$ & $3 \%$ & $75 \%$ \\
2010 & $\$$ & $16.124,69$ & $\$$ & 256,24 & $2 \%$ & $14 \%$ & $-40 \%$ \\
2011 & $\$$ & $19.791,13$ & $\$$ & 293,82 & $1 \%$ & $23 \%$ & $15 \%$ \\
2012 & $\$$ & $22.253,15$ & $\$$ & 403,44 & $2 \%$ & $12 \%$ & $37 \%$ \\
2013 & $\$$ & $26.889,38$ & $\$$ & 346,48 & $1 \%$ & $21 \%$ & $-14 \%$ \\
2014 & $\$$ & $29.813,56$ & $\$$ & 429,99 & $1 \%$ & $11 \%$ & $24 \%$ \\
2015 & $\$$ & $27.373,25$ & $\$$ & 452,07 & $2 \%$ & $-8 \%$ & $5 \%$ \\
\hline
\end{tabular}

Fuente: Banco Central Del Ecuador (Bce) / Boletín Anuario Nro. 38

El gasto público ecuatoriano ha vivido momentos de alza positivos, en el 2008 tuvo un incremento del $38 \%$, siendo el incremento registrado más alto de la última década, teniendo una relación con la subida del precio del barril de petróleo el cual paso de 70.50 USD a 97.90 USD para la exportación, lo que mejoró los ingresos del Estado y por ende se asignó un mayor monto presupuestado para el gasto público, es mencionado año un punto crítico alto sobre el cual se ha mantenido un incremento porcentual promedio del $14 \%$ dentro del periodo de estudio, a excepción del año 2015 el cual se contrajo en un 8\% (Tabla $\mathrm{N}^{\circ} 1$; Figura $\mathrm{N}^{\circ} 1$ ).

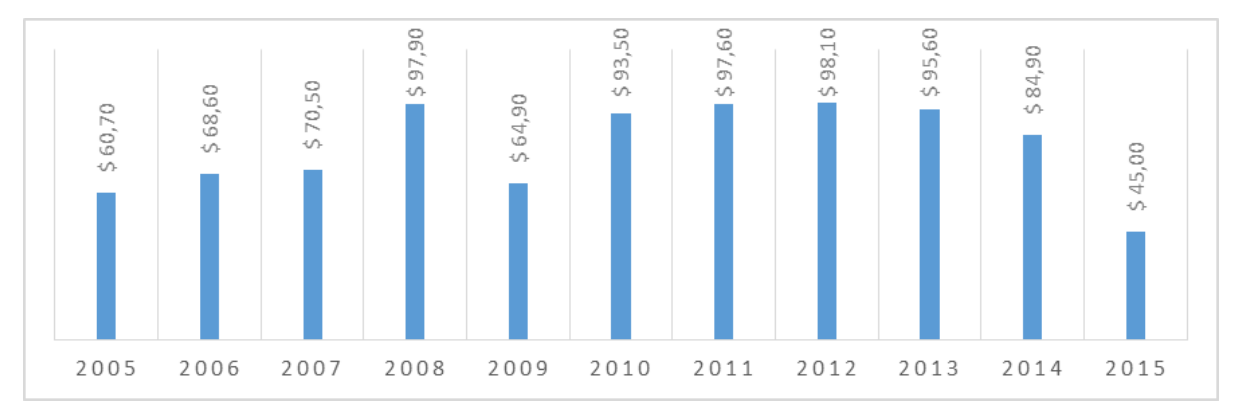

Fig. 1. Evolución De Los Precios Promedio Del Crudo Ecuatoriano Por Barril. (2005-2015) 
Fuente: Banco Central Del Ecuador (BCE)

Mencionado año presento una variación porcentual del gasto público que registra un valor negativo guardando una correlación con la caída del precio promedio del crudo ecuatoriano. La política estatal jugo un rol importante frente a la caída del precio del barril de petróleo y la apreciación del dólar frente al euro, el gobierno aplicó medidas restrictivas que redujeron el gasto público convirtiendo a los tributos y otros ingresos no petroleros en la principal fuente de financiamiento del presupuesto, para evitar un decrecimiento económico y un ambiente de incertidumbre (Rivera, 2013) (Tabla $N^{\circ} 1$; Figura $N^{\circ} 1$ ).

En el año 2008 y 2009 el sector agropecuario del país obtuvo el más importante incremento en términos de recursos monetarios otorgados por el Estado. El incremento registrado en el gasto público agrícola del periodo 2008 de un $98,59 \%$ y del 2009 de un $75 \%$ es de orden superior y antagónico al decrecimiento que se sostuvo el 2006 y el 2007, mencionado comportamiento es similar al mostrado en el precio promedio del barril de petróleo (Tabla $\mathrm{N}^{\circ} 1$ ).

En el 2010 se registra un decrecimiento del -40\%, según datos proporcionados por el BCE (2016), esto provocado por la asignación prioritaria a sectores como educación, transporte, sueldos, recursos naturales y energéticos, y deuda pública. Para los años 2011 y 2012 el presupuesto del gasto público destinado al sector agropecuario subió en un 57\%, tras una reasignación del presupuesto, ajustando a otros sectores estratégicos y por el aumento de gasto público (Tabla $\mathrm{N}^{\circ} 1$ ).

El gasto en desarrollo agropecuario manifiesta una pendiente negativa con un decrecimiento de $-14 \%$ para el año 2013, para este periodo se impulsaron proyectos como la construcción de hidroeléctricas, colegios del milenio, etc., reajustando el presupuesto de mencionado sector para beneficio de otros, comportamiento contradictorio con lo registrado en los años 2014 y 2015 con un incremento del 24\% y 5\% respectivamente impulsando proyectos para la población rural, en base al cambio de la matriz productiva y el desarrollo del sector, a pesar de la caída del precio del barril de petróleo (Tabla $\mathrm{N}^{\circ} 1$; Figura $\mathrm{N}^{\circ} 1$ ).

A diferencia de lo mencionado él PIB presenta una tendencia positiva constante, teniendo un incremento del 2005 al 2015 del 47\%. Durante el periodo de estudio el PIB Agrícola ha registrado un incremento positivo constante pero leve con un valor porcentual de variación promedio del 3,9\% manteniendo un aporte al PIB total promedio del 8.9\% (Tabla $\mathrm{N}^{\circ} 2$ ).

Tabla 2. Participación Del Producto Interno Bruto Agrícola Dentro Del Producto Interno Bruto Total. (2005-2015) 


\begin{tabular}{cccccccc}
\hline \multicolumn{7}{c}{ PIB miles de dólares de 2007 } \\
\hline EJERCICIO & & PIB 2005 - 2015 & $\begin{array}{c}\text { PIB (Agricultura; acuicultura } \\
\text { y pesca de camarón; pesca } \\
\text { excepto camarón) } \\
\text { 2015 - }\end{array}$ & $\begin{array}{c}\text { Participación } \\
\text { Agropecuaria en el } \\
\text { PIB }\end{array}$ & $\begin{array}{c}\text { Variación anual del } \\
\text { PIB total }\end{array}$ & $\begin{array}{c}\text { Variación anual del } \\
\text { PIB Agropecuario }\end{array}$ \\
\hline 2005 & $\$$ & 47.809 .319 & $\$$ & 4.423 .717 & $9,25 \%$ & - & - \\
2006 & $\$$ & 49.914 .615 & $\$$ & 4.614 .324 & $9,24 \%$ & $4,40 \%$ & $4,31 \%$ \\
2007 & $\$$ & 51.007 .777 & $\$$ & 4.772 .112 & $9,36 \%$ & $2,19 \%$ & $3,42 \%$ \\
2008 & $\$$ & 54.250 .408 & $\$$ & 4.851 .668 & $8,94 \%$ & $6,36 \%$ & $1,67 \%$ \\
2009 & $\$$ & 54.557 .732 & $\$$ & 4.934 .884 & $9,05 \%$ & $0,57 \%$ & $1,72 \%$ \\
2010 & $\$$ & 56.481 .055 & $\$$ & 4.971 .366 & $8,80 \%$ & $3,53 \%$ & $0,74 \%$ \\
2011 & $\$$ & 60.925 .064 & $\$$ & 5.366 .155 & $8,81 \%$ & $7,87 \%$ & $7,94 \%$ \\
2012 & $\$$ & 64.362 .433 & $\$$ & 5.407 .764 & $8,40 \%$ & $5,64 \%$ & $0,78 \%$ \\
2013 & $\$$ & 67.546 .128 & $\$$ & 5.769 .740 & $8,54 \%$ & $4,95 \%$ & $6,69 \%$ \\
2014 & $\$$ & 70.243 .048 & $\$$ & 6.025 .854 & $8,58 \%$ & $3,99 \%$ & $4,44 \%$ \\
2015 & $\$$ & 70.353 .852 & $\$$ & 6.256 .473 & $8,89 \%$ & $0,16 \%$ & $3,83 \%$ \\
\hline
\end{tabular}

Fuente: Banco Central Del Ecuador / Boletín Anuario Nro. 38

Los periodos con registros de importante crecimiento por parte del PIB Agropecuario se dieron en el año 2011, el cual presento un incremento del 7.94\%, mientras que, en el año 2013, este ascendió a $6.69 \%$, demostrando la importancia del sector en el desarrollo económico del país (Tabla $\mathrm{N}^{\circ} 2$ ).

El Estado ecuatoriano invirtió en el desarrollo productivo del sector agropecuario el 2\% promedio del gasto público total durante el periodo 2005-2015, mencionado valor porcentual es relativamente inferior frente al brindado a otros sectores económicos. El IOA pretende evaluar el interés que tienen los estados al momento de invertir en los sectores productivos tomando en cuenta la contribución que hacen estos para la economía de sus países, para el caso ecuatoriano este indicador muestra un escaso aporte del Ecuador a la agricultura en términos de gasto publico teniendo un promedio del 0,19 dentro de la década de estudio, sin acercarse a la unidad entendiendo que los gobiernos deben ser recíprocos con la contribución que generan los sectores productivos (Tabla $\mathrm{N}^{\circ} 3$; Figura $\mathrm{N}^{\circ}$ ).

Este indicador demuestra que los gobiernos ecuatorianos que conformaron el periodo de estudio han presentado poco interés en invertir en el desarrollo productivo del sector agropecuario entendiendo a este como el aporte porcentual que genera el gasto público al sector en contraste del aporte porcentual que contribuye mencionado sector a la economía del país. El 2009 presenta el mayor valor registrado dentro del periodo de estudio con un 0,33 ; valor distante de la unidad (Tabla $\mathrm{N}^{\circ} 3$; Figura $\mathrm{N}^{\circ} 2$ ).

Cabe destacar que mencionado indicador se limita a evaluar el aporte del Estado en términos porcentuales. Dentro del periodo de estudio se generó un incremento del gasto público total lo cual en términos de unidad monetaria presento un notable incremento, pero que en términos de participación del mismo frente al resto de sectores su contribución es menor (Tabla $\mathrm{N}^{\circ}$; ; Figura $\mathrm{N}^{\circ}$ 2).

Tabla 3. Índice De Orientación Agrícola. (2005-2015) 


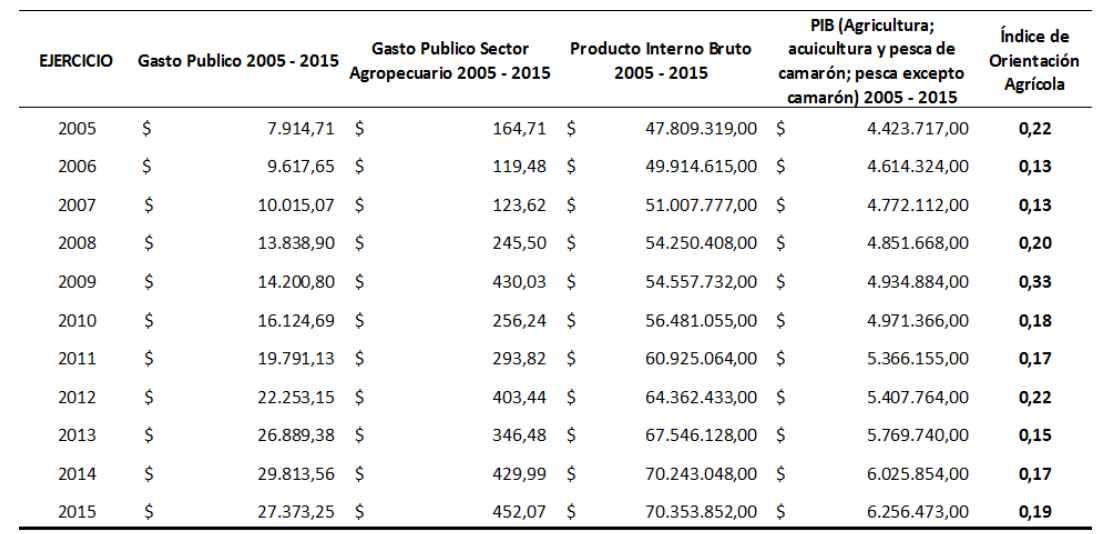

Fuente: Banco Central Del Ecuador / Boletín Anuario Nro. 38

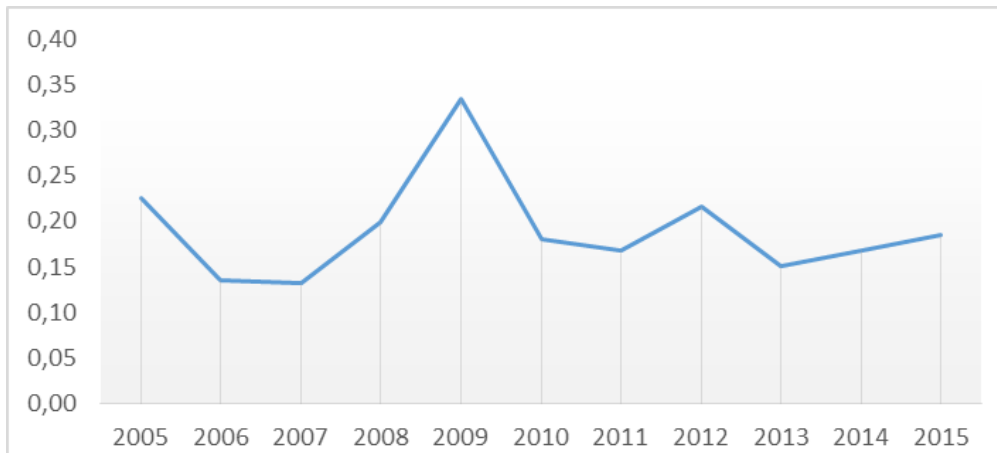

Figura 2. Comportamiento Del Índice De Orientación Agrícola. (2005-2015) Fuente: Banco Central Del Ecuador (BCE)

El comportamiento del gasto público total marca una tendencia positiva, hasta el año 2015 donde tuvo un decrecimiento el cual encuentra relación con el precio del barril de petróleo, tal como lo menciona Quinde (2011) este incremento del gasto público se obtiene por los altos ingresos petroleros, el decrecimiento existente en los egresos del Estado se da por la caída del precio del barril de crudo ecuatoriano a partir del año 2015.

El gasto público del sector agropecuario muestra una tendencia irregular decreciendo en los años 2010 y 2013, ante este comportamiento Quinde (2011) manifiesta que la otorgación de recursos para el sector agropecuario es mal manejada, impidiendo de cierta manera el desarrollo productivo del sector, estando de acuerdo al momento de aseverar que el Estado ha duplicado el presupuesto de sectores sin un estudio previo, específicamente sin cubrir necesidades para el sector agropecuario del país.

El aporte que genera el sector agropecuario al PIB se mantiene al alza dentro del periodo de estudio, confirmando que a pesar del poco gasto público y la falta de inversión privada la agricultura es uno de los principales motores productivos y económicos del Ecuador, esta premisa discrepa con lo mencionado por Quinde (2011) al momento de mencionar que la falta de interés se ve reflejada en el PIB agropecuario.

El comportamiento del IOA en Ecuador presenta una tendencia irregular, confirmando lo mencionado por la Organización de las Naciones Unidas para la Alimentación y la Agricultura FAO (2012) donde afirma que, para el Estado, el sector agropecuario no es un eje prioritario para 
él desarrollo, presentando un promedio de tan solo 0,19 a diferencia de países como México y Brasil (BM, 2009).

Quinde (2011), corrobora dicho comportamiento mencionando que el Ecuador es uno de los países con menor gasto público para el sector agropecuario, sin tomar importancia de la participación del sector agropecuario a la producción interna nacional, esto da como resultado una otorgación de recursos mal manejada, sin base ni sustento con estudios del caso, beneficiando a otros sectores económicos del país.

\section{Conclusiones}

El Gasto Publico Nacional ha sido duplicado por el gobierno sin un estudio previo gracias al alza de ingresos petroleros, beneficiando a sectores de la economía en el incremento de gastos administrativos innecesarios, como sueldos de servidores públicos, descuidando el desarrollo agropecuario del país. El gobierno tuvo que reducir un $8 \%$ el Gasto público ante la reducción de los ingresos, provocada por la caída del precio de barril de petróleo, pero el gasto público agropecuario presento un ligero incremento, ya que el gobierno mantiene la iniciativa para el cambio de matriz productiva, a pesar de esto mencionada asignación se considera no relevante con la contribución del sector a la economía ecuatoriana.

El aporte que genera el sector agropecuario al PIB sigue siendo representativo, manteniendo una participación promedio del $8.9 \%$ anual, aun sabiendo que la agricultura ha sido relegada por parte del Gobierno, con un gasto publico agropecuario de tendencia irregular y de poca participación. El sector agropecuario a pesar de la poca asignación del gasto público, su participación en el PIB nunca decreció como se puede constatar en el presente documento, esto se puede presentar como una oportunidad para manifestar la necesidad de otorgar mayores recursos para dicho sector.

\section{Bibliografía}

Arias, Joaquín; Vallejo, Silvana y Trejos, Rafael. 2005. La Real Contribución de la Agricultura a la Economía del Ecuador. Ecuador: Instituto Interamericano de Cooperación para la Agricultura - IICA.

Bacon, Francis. 1949. Novum Organum. Buenos Aires: Losada. (Versión Original 1620)

Barcelata, Hilario. 2007. Las Finanzas Públicas En México. 1988-2006. México: Eumed

BCE, Banco Central del Ecuador. 2016. Boletín Anuario Número 38. Quito.

BM, Banco Mundial. 2009. Análisis del Gasto Público en el Desarrollo Agrícola y Rural. México.

Duque, Edison. 2015. Seminario Metodología de la Investigación. Colombia: Universidad Nacional de Colombia

Echeverri, Rafael y Ribero, María. 2002. Nueva ruralidad: visión del territorio en América Latina y el Caribe. San José: Instituto Interamericano de Cooperación para la Agricultura - IICA 
FAO, Organización de las Naciones Unidas para la Alimentación y la Agricultura. 2004. Política de desarrollo agrícola. Roma

FAO, Organización de las Naciones Unidas para la Alimentación y la Agricultura. 2007. Análisis Integral Del Gasto Público Agropecuario. México

FAO, Organización de las Naciones Unidas para la Alimentación y la Agricultura. 2012. El Estado Mundial de la Agricultura y la Alimentación. Roma.

Guereña, Arantxa. 2011. Derecho a producir. Invertir más y mejor en la pequeña agricultura de América del sur. Mexico: Oxford Committee for Famine Relief - Oxfam

Hall, David. 2010. Por qué es necesario el gasto público. Reino Unido: Public Services International PSI, Business School, University of Greenwich

Hirschman, Albert. 1958. The Strategy of Economic Development. New Haven: Yale University Press.

INEC, Instituto Nacional de Estadística y Censo. 2017. Encuesta Nacional de Empleo, Desempleo y Subempleo. Quito

MAGAP, Ministerio de Agricultura, Ganadería, Acuacultura y Pesca. 2016. La política agropecuaria ecuatoriana: hacia el desarrollo territorial rural sostenible: 2015-2025 I Parte, ISBN: 978-994222-019-6. Quito

MAGAP, Ministerio de Agricultura, Ganadería, Acuacultura y Pesca. 2016. La política agropecuaria ecuatoriana: hacia el desarrollo territorial rural sostenible: 2015-2025 II Parte, ISBN: 9789942-22-019-6. Quito

Martínez Valle, Luciano. 2009. La pluriactividad entre los pequeños productores rurales: el caso ecuatoriano. En La pluriactividad en el campo latinoamericano (1st ed., pp. 81-101). Quito: FLACSO, Facultad Latinoamericana de Ciencias Sociales.

Naranjo, Marcos. 2009. Un Puerto en busca de una Nación, Guayaquil y la idea fundacional del Ecuador como país. Ecuador: Facultad Latinoamericana de Ciencias Sociales - FLACSO. Seminario Internacional Poder, Política y Repertorios de la Movilización Social en el Ecuador Bicentenario

Quinde-Rosales, Victor. 2011. Evolución del Gasto Público Agropecuario y Rural en el Ecuador Periodo 2000-2009. Ecuador: Universidad Agraria del Ecuador - UAE

Quinde-Rosales, Victor. 2015. Efecto de la Intervención Estatal en la Regulación de los Precios del Arroz Cantón Daule. Ecuador: Universidad Agraria del Ecuador - UAE

Rivera, Patricio. 2013. Ecuador está preparado para enfrentar una posible crisis. Revista Ecuador Económico. Quito: MCPE, Ministerio Coordinador de la Política Económica.

Yannuzzelli, Jean Pierre. 2017. El Índice de Orientación Agrícola (IOA) como Mecanismo del Análisis del Gasto Público Agropecuario Ecuatoriano. Período 2005 - 2015. Ecuador: Universidad Agraria del Ecuador - UAE 